\title{
Optimization of grazing incidence mirrors and its application to surveying $\mathrm{X}$-ray telescopes
}

\author{
P. Conconi ${ }^{1}$ and S. Campana ${ }^{1}$ \\ Osservatorio Astronomico di Brera, Via Bianchi 46, 23807 Merate (Lc), Italy
}

Received 15 November 2000 / Accepted 30 March 2001

\begin{abstract}
Grazing incidence mirrors for X-ray astronomy are usually designed in the parabola-hyperbola (Wolter I) configuration. This design allows for optimal images on-axis, which however degrade rapidly with the off-axis angle. Mirror surfaces described by polynomia (with terms higher than order two), have been put forward to improve the performances over the field of view. Here we present a refined procedure aimed at optimizing wide-field grazing incidence telescopes for X-ray astronomy. We improve the angular resolution over existing (wide-field) designs by $\sim 20 \%$. We further consider the corrections for the different plate scale and focal plane curvature of the mirror shells, which sharpen by another $\sim 20 \%$ the image quality. This results in a factor of $\sim 2$ reduction in the observing time needed to achieve the same sensitivity over existing wide-field designs and of $\sim 5$ over Wolter I telescopes. We demonstrate that such wide-field X-ray telescopes are highly advantageous for deep surveys of the $\mathrm{X}$-ray sky.
\end{abstract}

Key words. telescopes - surveys - X-rays: general

\section{Introduction}

Imaging the X-ray sky has become possible thanks to grazing incidence telescopes. These telescopes (the first flew on the Einstein observatory; VanSpeybroeck 1979; Giacconi et al. 1979) made possible a great improvement in sensitivity and angular resolution, allowing for the study of virtually all classes of X-ray sources. Telescopes on board Einstein, EXOSAT and ROSAT mainly covered the low energy band $(\lesssim 4 \mathrm{keV})$, whereas the production of very smooth gold surfaces made possible the imaging up to $\sim 10 \mathrm{keV}$ with BBXRT (Serlemitsos et al. 1992), ASCA (Serlemitsos et al. 1995) and BeppoSAX (Citterio et al. 1991).

Two main strategies have been adopted to further develop X-ray imaging: $i$ ) increasing the angular resolution, reaching an on-axis values rivaling optical images $\left(\sim 0.5^{\prime \prime}\right)$, as in the case of Chandra (Weisskopf et al. 2000); ii) increasing the effective area in order to collect a larger number of photons (e.g. for spectral purposes) but with a poorer angular resolution, as in the case of Newton-XMM (Jansen et al. 2000). Proposed X-ray missions move along these lines: MAXIM is expected to reach 100 microarcsec (at least; Cash et al. 2000) whereas on the other side Constellation- $X$ is expected to increase the effective area $\left(14500 \mathrm{~cm}^{-2}\right.$ at $\left.1 \mathrm{keV}\right)$ with an angular

Send offprint requests to: S. Campana,

e-mail: campana@merate.mi.astro.it resolution of $\sim 20$ arcsec (Tananbaum et al. 1999). Finally, XEUS will increase dramatically the effective area (up to $300000 \mathrm{~cm}^{-2}$ ) with a good angular resolution of $\lesssim 5^{\prime \prime}$ (Bavdaz et al. 1999).

$\mathrm{X}$-ray astronomy benefited from serendipitous surveys (e.g. Einstein EMSS, Gioia et al. 1990; EXOSAT HGSC, Giommi et al. 1991; ROSAT WGA, White et al. 1994; ROSAT SRC, Zimmermann 1994; BMW ROSHRI, Campana et al. 1998; ROSHRICAT, Voges et al. 1999a; ASCA SIS, Gotthelf \& White 1997). The first all-sky survey with an imaging $\mathrm{X}$-ray telescope was carried out by ROSAT, spending its first 6 months surveying the entire sky. The ROSAT All-sky Survey (RASS, 0.1-2.4 keV; Voges et al. 1999b, 2000) is now an extremely useful tool for all kinds of astronomers.

As a pathfinder for new missions and to further improve the statistical studies of fainter/harder sources, new all-sky surveying missions are under study, such as ABRIXAS (Trümper et al. 1998) WAXS-WFXT/Panoram-X (Chincarini et al. 1999, 2000).

Mirrors are usually built in the Wolter I (paraboloidhyperboloid) configuration (Wolter 1952a, 1952b) which provides perfect images on-axis in principle. This design exhibits no spherical aberration but suffers from field curvature, coma and astigmatism, which make the angular resolution degrade rapidly with increasing off-axis angles (VanSpeybroeck \& Chase 1972). Recently, Harvey \& Thompson (1999) put forward the idea of a telescope made 
Table 1. Grazing incidence mirror designs.

\begin{tabular}{ccccc}
\hline Name & $a_{2}$ & $b_{2}$ & $a_{3}$ & $b_{3}$ \\
\hline Parabolic & 0 & 0 & 0 & 0 \\
Dbl.-cone & $\tan ^{2} \alpha$ & $\tan ^{2} \beta$ & 0 & 0 \\
Wolter I & 0 & $2 \rho_{0} \tan \beta /\left(z_{0}+\rho_{0} \cot 2 \alpha\right)$ & 0 & 0 \\
\hline
\end{tabular}

by two hyperboloid surfaces (see also Nariai 1987, 1988) which provides good performances over a field of $\sim 20^{\prime}$ and was adopted for the Solar X-ray Imager telescope.

More general mirror designs than Wolter's exist in which the primary and secondary mirror surfaces are expanded as a power series (Werner 1977; double-cone and Wolter I profiles are described by low order coefficients, less than two). These polynomial solutions are well suited for optimization purposes, which may be used to increase the angular resolution at large off-axis positions, degrading the on-axis performances. The idea is to transfer the principle of the Ritchey-Chrétien Cassegrein telescope, widely used in optical astronomy, to grazing incidence optics. By deliberately compromising the on-axis performances, one can introduce aberrations (mainly spherical) that tend to cancel or reduce the off-axis aberrations.

Burrows et al. (1992; BBG hereafter) discuss polynomial optics solutions (Werner 1977) and described in detail a wide-field $\mathrm{X}$-ray telescope with good angular resolution up to $\sim 30^{\prime}$. This new approach was also used to describe an optimization of the Chandra performances for survey work. Here we improve the BBG's optimization technique and sketch a procedure aimed at optimizing the mirror assembly. We further point out the need for an all-sky survey deeper and at energies higher than the ROSAT's. This relies on science (especially cosmology) and on the lack of a pathfinder for the $\mathrm{X}$-ray missions of the next generation, such as XEUS and Constellation- $X$.

\section{Optical design}

The X-ray telescopes we consider here (see BBG for a more general view) are made by two coaxial surfaces of revolution, which intersect at a circle, called the intersection plane (IP). A set of parameters characterize the mirror surfaces: the radius of the mirror shell at the IP, $\rho_{0}$; the telescope focal length, $z_{0}$; the angle, $\alpha(\beta)$, between the primary (secondary) mirror tangent at the IP and the optical axis; the length of the primary (secondary) mirror, $Z_{1}\left(Z_{2}\right)$. A shell scheme is depicted in Fig. 1, with the origin of the Cartesian system at the IP and the $z$ axis along the optical axis of the telescope to the source.

In this reference system the primary and secondary mirror surfaces can be expanded as a power series of the form

$$
\frac{\rho_{1}^{2}}{\rho_{0}{ }^{2}}=\sum_{i=0}^{n_{1}} a_{i}\left(\frac{z_{1}}{\rho_{0}}\right)^{i} \quad \frac{\rho_{2}^{2}}{\rho_{0}^{2}}=\sum_{i=0}^{n_{2}} b_{i}\left(\frac{z_{2}}{\rho_{0}}\right)^{i}
$$

where $\left(\rho_{1}, z_{1}\right)$ and $\left(\rho_{2}, z_{2}\right)$ are radial and axial coordinates of the primary and secondary surfaces, respectively.
$\mathrm{X}$-ray telescopes provide the best collecting area for a given total mirror length (and also the best reflection efficiency at short wavelengths for a given diameter to focal length ratio) in the case of $\xi=\alpha /(\beta-2 \alpha)=1$ (VanSpeybroeck \& Chase 1972). Thus, the highest reflection efficiency is achieved for $\beta=3 \alpha$ and a focal length $z_{0}=\rho_{0} /(\tan 4 \alpha)$. By definition $a_{0}=b_{0}=1$, while $a_{1}=-2 \tan \alpha$ and $b_{1}=-2 \tan \beta$ are twice the slope of the primary and the secondary surfaces at IP. By selecting different values for the other coefficients one obtains different optical designs (see Table 1).

\section{Optimizing single mirror shells}

Simple recipes have been proposed and used in building $\mathrm{X}$-ray telescopes to improve the image quality over the full field of view without affecting the mirror surfaces. The simplest recipe consists of slightly defocussing the optical system (e.g. Cash et al. 1979). This suggestion was adopted for the flight module of the JET-X telescopes, displacing the focal plane from the nominal value by $-2.5 \mathrm{~mm}$. A different (and better) approach consists of tilting the detector so as to follow the (curved) focal plane. This configuration has been used for the 4 CCD detectors of the ACIS-I camera on board Chandra, which were assembled in an inverse shallow pyramid configuration. A similar approach has been adopted for the 7 CCDs of the MOS camera: the central CCD is at the focal point on the optical axis while the outer six are stepped towards the mirror by $4.5 \mathrm{~mm}$ to follow approximately the focal plane curvature (Turner et al. 2001).

Working directly on the mirror properties provides a more powerful tool to improve the image quality over the field of view. Polynomial surfaces are particularly well suited for optimizing purposes, since computing procedures can operate iteratively on the coefficients of the power series expansion. This can be done by defining a merit function and by finding its minimum in the coefficient parameter space, after specifying a minimization goal. These criteria can either provide the best image onaxis with only a modest improvement off-axis (i.e. compromising the on-axis performances by a given fraction), or the flattest response over the entire field of view (see also BBG). In this paper we focus on the latter problem, having in mind $\mathrm{X}$-ray instruments dedicated to $\mathrm{X}$-ray surveys ${ }^{1}$. The key ingredients to make up an appropriate merit function are: $i$ ) an indicator of the mirror image quality at different off-axis positions $I(\theta)$ (e.g. spot rms, Half Energy Width, HEW, or more generally a fixed value of the Encircled Energy Function, EEF) and $i i$ ) a weighting function $W(\theta)$. If manufacturing errors and alignment tolerances can be summed up in quadrature to $I(\theta)$, they

\footnotetext{
1 ROSAT mirrors have been optimized under a number of constraints (e.g. Wolter I geometry) to carry out an all-sky survey (Aschenbach 1988).
} 


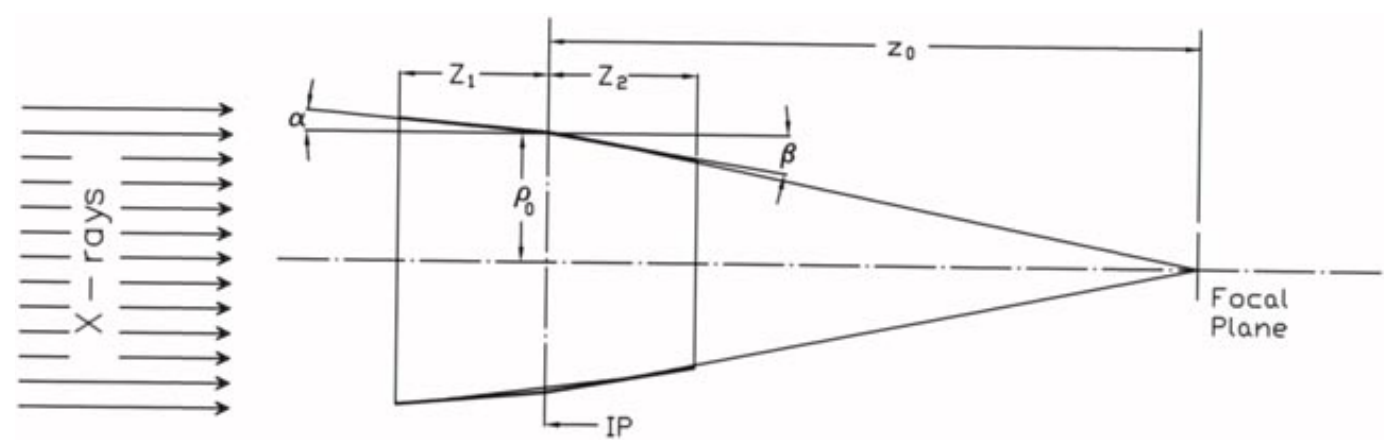

Fig. 1. Telescope configuration and parameters.

will not alter the location of the minimum. As a general rule, the merit function can be expressed as:

$M=\int_{0}^{\theta_{0}} I(\theta) W(\theta) \mathrm{d} \theta \simeq \sum_{i=0}^{N} I\left(\Theta_{i}\right) W\left(\Theta_{i}\right)$

where $N$ is a number of the fixed positions $\Theta_{i}$ where the image quality has to be evaluated.

The minimization of the merit function is usually carried out through ray-tracing simulations which accounts for the mirror properties: the image quality function $I(\theta)$ is computed for a given number of off-axis positions together with the weights $W(\theta)$ and the resulting merit function is evaluated for a specific value of the mirror parameters $\left\{a_{j} ; b_{j}\right\}$. Minimization is then carried out by varying the values of the $\left\{a_{j} ; b_{j}\right\}$ parameters.

As a working example, we describe the optimization of an $\mathrm{X}$-ray telescope with a $\sim 30^{\prime}$ radius field of view. We consider the same outer shell as in BBG (see Table 2 for mirror characteristics) and optimize it with the following prescriptions. The merit function we consider is:

$M=\sum_{j=0}^{n} \sum_{i=1}^{m} d_{i}^{2}\left(\Theta_{j}\right) \times\left[A_{\mathrm{eff}}\left(\Theta_{j}, E\right) \times W\left(\Theta_{j}\right)\right]$

where $n$ is the number of off-axis angles over which the merit function is evaluated and $m$ the number of photons used in the ray-tracing simulation at each position. $d_{i}$ is the distance between the position of the $i$ th photons from the center of gravity of the image at $\Theta_{j}$. Unlike BBG (who adopted the spot $\mathrm{rms}$ ), we consider a given fraction of the encircled energy (after positioning the spot center of gravity); in particular we considered the $50 \%$ (HEW) and $80 \%$ EEF. This gives a more realistic description of the image quality in view of the detection of point and extended sources. $A_{\text {eff }}$ is the effective area at a given off-axis angle (i.e. the vignetting function) at a given energy $E$ and allows us to optimize the mirrors where more photons are collected. A second weight is represented by the function $W$ defined as

$W\left(\Theta_{j}\right)= \begin{cases}\Theta_{j}+\Theta_{j+1} & 0 \leq j<n \\ \Theta_{n} & j=n\end{cases}$

which allows us to properly weight the growing area at large off-axis angles (in the case of equidistant sampling
Table 2. WFT mirror shell characteristics.

\begin{tabular}{cccc}
\hline Parameter & Outer shell & Median shell & Inner Shell \\
\hline Focal distance & $3000.0 \mathrm{~mm}$ & $3003.3 \mathrm{~mm}$ & $3005.5 \mathrm{~mm}$ \\
Axial shift at IP & $0.0 \mathrm{~mm}$ & $3.4 \mathrm{~mm}$ & $5.7 \mathrm{~mm}$ \\
Mirror radius at IP & $300.0 \mathrm{~mm}$ & $271.1 \mathrm{~mm}$ & $239.2 \mathrm{~mm}$ \\
Maximum radius & $303.0 \mathrm{~mm}$ & $273.5 \mathrm{~mm}$ & $241.0 \mathrm{~mm}$ \\
Minimum radius & $291.0 \mathrm{~mm}$ & $263.9 \mathrm{~mm}$ & $233.8 \mathrm{~mm}$ \\
Mirror length $^{*}$ & $120 \mathrm{~mm}$ & $120 \mathrm{~mm}$ & $120 \mathrm{~mm}$ \\
Scaled length $^{*}$ & $120 \mathrm{~mm}$ & $100 \mathrm{~mm}$ & $85.3 \mathrm{~mm}$ \\
\hline$a_{2} \times 10^{-3}$ & 0.87 & 0.69 & 0.42 \\
$b_{2} \times 10^{-3}$ & 3.99 & 3.31 & 2.78 \\
$a_{3} \times 10^{-3}$ & 2.34 & 2.01 & 1.39 \\
$b_{3} \times 10^{-3}$ & 3.40 & 2.53 & 0.91 \\
$a_{4} \times 10^{-3}$ & 3.35 & 3.33 & 3.11 \\
$b_{4} \times 10^{-3}$ & -6.45 & -4.57 & -0.81 \\
$a_{5} \times 10^{-3}$ & 1.47 & 1.68 & 2.59 \\
$b_{5} \times 10^{-3}$ & 5.15 & 3.48 & 0.04 \\
\hline Spot rms $(\mathrm{BBG})^{\dagger}$ & 3.03 & 2.92 & 3.92 \\
Spot rms & 2.76 & 2.59 & 2.77 \\
\hline
\end{tabular}

* Length of the primary (or secondary) mirrors.

${ }^{\dagger}$ Evaluated on the best tilted focal plane.

angles). Finally, we consider fifth order polynomia (rather than the third order of BBG) and make sure that the inclusion of additional terms do not improve the minimization.

For each off-axis angle we evaluated the image characteristics at the best focal position, i.e. following a curved focal plane. In particular, we chose the curved focal plane which maximizes the $80 \% \mathrm{EEF}$ (see Fig. 2), which is slightly different from the one individuated by the HEW. The profile we obtained gives better results than BBG's especially at small off-axis angles where the mirror effective area is larger and the mean HEW and $80 \%$ EEF over the whole field of view are about $20 \%$ better than in the BBG design (see Table 3). Because curved CCD X-ray detectors do not exist, more realistic results can be obtained by considering an approximation of the curved focal plane obtained with a tilted configuration (as described for the Chandra satellite, see above). We evaluated the mirror performances on the best tilted focal plane (tilt of about 3 degrees). Also in this configuration, we obtained a mean HEW and $80 \%$ EEF improving by a factor of $~ 20 \%$ BBG's design (see Table 3). 
WFXT single mirror

Best tilted focal plane

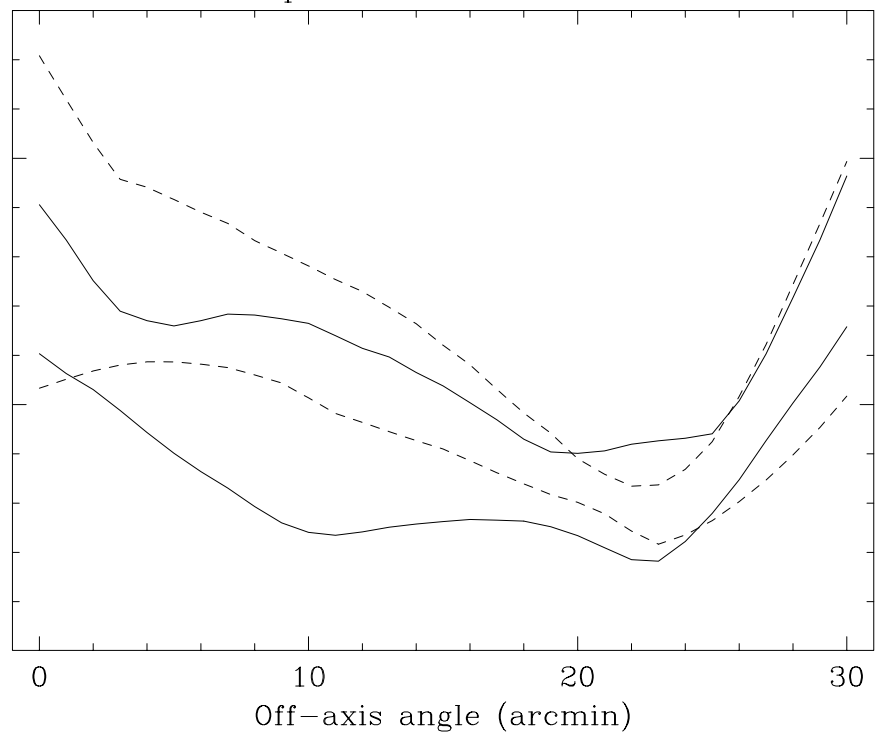

Fig. 2. HEW and $80 \%$ EEF for different mirror designs as a function of the off-axis angle for the outermost shell of Table 2. The dashed lines correspond to the BBG design and the continuous lines to our best profile. Of the two lines for each mirror design, the upper one refers to the $80 \% \mathrm{EEF}$ and the lower to the HEW. These values are estimated at the best focal position for each off-axis angle.

Table 3. Mean HEW and $80 \%$ EEF over the field of view for the largest mirror shell of the WFT.

\begin{tabular}{c|cc|cc}
\hline $\begin{array}{c}\text { Mirror } \\
\text { type }\end{array}$ & $\begin{array}{c}\text { Mean } \\
\text { HEW } \\
\text { (arcsec) }\end{array}$ & $\begin{array}{c}\text { Mean } \\
80 \% \text { EEF } \\
\text { (arcsec) }\end{array}$ & $\begin{array}{c}\text { Mean } \\
\text { HEW } \\
\text { (arcsec) }\end{array}$ & $\begin{array}{c}\text { Mean } \\
80 \% \text { EEF } \\
\text { (arcsec) }\end{array}$ \\
\hline Wolter I & 7.0 & 11.4 & 7.1 & 11.6 \\
BBG & 3.2 & 5.2 & 4.0 & 6.3 \\
$\mathrm{CC}^{\dagger}$ & 2.7 & 4.4 & 3.1 & 5.4 \\
\hline
\end{tabular}

The mean has been obtained by weighting the relevant angular response with the corresponding area of the field of view.

The first two columns on the HEW refer to the best focal position at each off-axis angle (i.e. curved focal plane), whereas the latest two refers to the best tilted plane.

$\dagger$ this work.

\section{Optimal design for wide-field $\mathrm{X}$-ray telescopes}

We consider here a simple version of a Surveying X-ray Telescope (SXT) consisting of three mirror shells, scaled from the largest one described in BBG, in order to deal with the mirror assembly. Each of these shells has been optimized separately, as described in the previous section. Mirror characteristics are reported in Table 2. The mean values for a confocal nesting are reported in Table 4 as CC-P. The HEW distribution as a function of the off-axis angle is plotted in Fig. 3 as the dotted line.

Images produced by the different mirror shells do not superpose exactly, having different plate scales. In particular, different shells focalize the relative image spots with an offset relative to one another that increases with the offaxis angle. This is a common problem of $\mathrm{X}$-ray telescopes

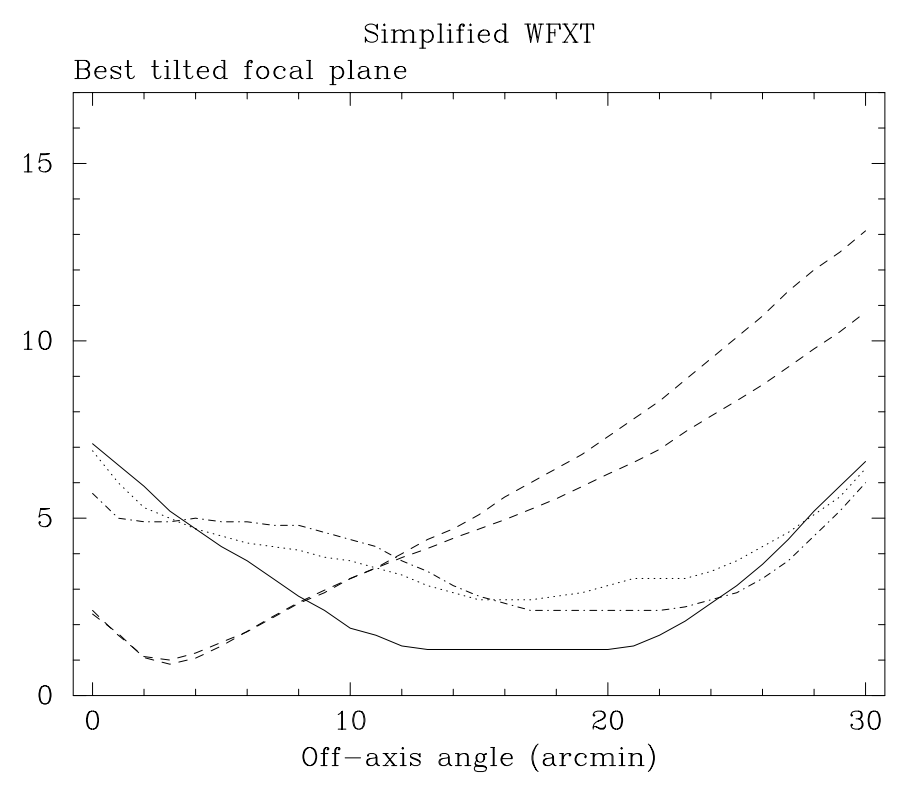

Fig. 3. HEW (in arcsec) for different mirror designs as a function of the off-axis angle, evaluated on the best tilted focal plane. The dotted line marks mirrors with a polynomial profile; the dot-dashed line mirrors optimized also for the plate scale and continuous line mirrors optimized also to have the same curvature of the focal plane. The dashed line represents the best HEW for a Wolter I telescope evaluated on the best tilted focal plane to maximize the response over the entire field of view. The upper dashed line is without corrections for plate scale and curvature, whereas the lower dashed line has these corrections included.

Table 4. Image characteristics for different mirror optimizations.

\begin{tabular}{c|ccc|cc}
\hline $\begin{array}{c}\text { Mirror } \\
\text { type }\end{array}$ & $\begin{array}{c}\text { Polyn. } \\
\text { opt. }\end{array}$ & $\begin{array}{c}\text { Plate } \\
\text { opt. }\end{array}$ & $\begin{array}{c}\text { Curvat. } \\
\text { opt. }\end{array}$ & $\begin{array}{c}\text { Mean } \\
\text { HEW } \\
\text { (arcsec) }\end{array}$ & $\begin{array}{c}\text { Mean } \\
80 \% \text { EEF } \\
\text { (arcsec) }\end{array}$ \\
\hline Wolter I & $\mathrm{N}$ & $\mathrm{N}$ & $\mathrm{N}$ & 7.5 & 12.3 \\
Wolter-PC & $\mathrm{N}$ & $\mathrm{Y}$ & $\mathrm{Y}$ & 7.0 & 11.9 \\
CC-P & $\mathrm{Y}$ & $\mathrm{N}$ & $\mathrm{N}$ & 3.9 & 7.9 \\
CC-PP & $\mathrm{Y}$ & $\mathrm{Y}$ & $\mathrm{N}$ & 3.6 & 6.8 \\
CC-PPC & $\mathrm{Y}$ & $\mathrm{Y}$ & $\mathrm{Y}$ & 2.8 & 5.3 \\
\hline
\end{tabular}

The mean has been obtained by weighting the relevant angular response with the corresponding area of the field of view. The HEWs and $80 \%$ EEFs have been evaluated on the best tilted focal plane.

and it results in an increase of the image blur at large offaxis angles. This problem can be overcome by making the mirror shells have different intersection planes, i.e. shells must be moved relative to one another (see also BBG). The resulting HEW distribution is plotted in Fig. 3 with a dashed line (see CC-PP in Table 4). The relative axial shifts at the IP are reported in Table 2 .

Mirrors shells of the same focal length have focal planes with different curvatures. This implies that for any offaxis angle the best focal plane is calculated by averaging over the shell best focal planes. On a tilted detector the problem is more severe and it introduces an image blur over the entire field of view. To overcome the problem one 


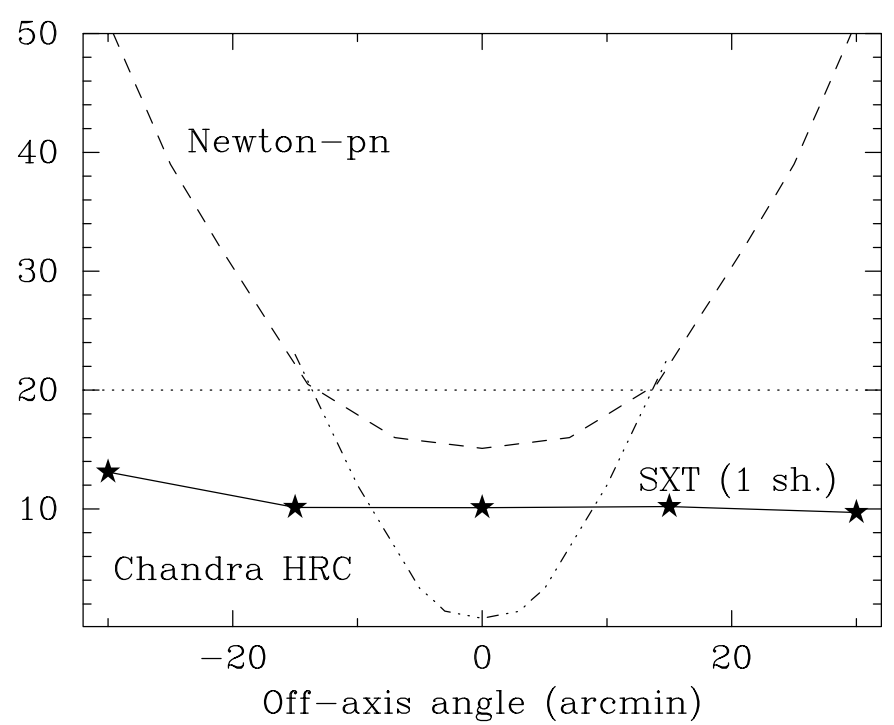

Fig. 4. Comparison of the HEW of the SXT mirror shell realized by Citterio et al. (1998; stars and continuous line) with current X-ray telescopes vs. the off-axis angle. A dot-dashed line marks Chandra-HRC and a dashed line Newton-pn instruments. The SXT HEW over the off-axis angle does not appear symmetric over the field due to a $-5^{\prime}$ mis-pointing in the calibrations (i.e. an off-axis angle of $-30^{\prime}$ corresponds to $-35^{\prime}$ ). A horizontal dotted line represents the $20^{\prime \prime}$ limit adopted in the text. Adapted from Citterio et al. (1998).

must build mirror shells which have the same best focal plane (i.e the same curvature). This can be achieved by constructing scaled versions of the outermost shell, implying that the innermost shells are shorter. The reduction of the inner shells results in a smaller effective area. This can be compensated by making the starting outermost shell longer.

Table 4 and Fig. 3 show the improved performance of our simple SXT. The polynomial design improves the mean HEW over the $30^{\prime}$ field of view by about $100 \%$ over the Wolter I design. The plate correction results in a small improvement $(\sim 10 \%)$, and the plate and curvature optimization (CC-PPC in Table 4) provide a gain of $\sim 40 \%$ over the polynomial design. In particular, the curvature optimization is more important and mirror performances are improved especially in the $10^{\prime}-20^{\prime}$ region (see Fig. 3, continuous line).

\section{Need for a wide-field telescope for all sky surveys}

In the infancy of X-ray astronomy several collimated experiments surveyed the $\mathrm{X}$-ray sky (e.g. Uhuru, OSO-7 and HEAO-1). The first imaging all-sky survey was conducted by ROSAT. The RASS has been extremely successful and now represents a powerful tool for X-ray astronomy (Voges et al. 1999b, 2000). The RASS revealed 18881 sources with a count rate higher than $0.05 \mathrm{c} \mathrm{s}^{-1}$ (comprising the Bright Source Catalog, BSC; Voges et al. 1999b) and 105924 sources with a likelihood ratio higher than 7
(Voges et al. 2000). Due to the scanning of great circles, the exposure times are highly variable from the equator to the ecliptic poles. A mean exposure time of $500 \mathrm{~s}$ can be considered (Voges et al. 1999b). Assuming a limit of $0.05 \mathrm{c} \mathrm{s}^{-1}$ for the survey (i.e. the same of the BSC) a rough flux limit of $5 \times 10^{-13} \mathrm{ergs}^{-1} \mathrm{~cm}^{-2}(0.1-2.4 \mathrm{keV})$ can be derived. The Position Sensitive Proportional Counter (PSPC) detector with which the RASS has been carried out allowed for a HEW of $\sim 20^{\prime \prime}$ on-axis and of $160^{\prime \prime}$ at 50 arcmin off-axis. A mean HEW of about $60^{\prime \prime}$ is expected for a scanining trace passing from the center (and worse for the others). The resulting positional accuracy is for sources in the BSC at a level of $25^{\prime \prime}$ (90\% confidence level; Voges et al. 1999b).

The need for an all-sky survey at high energies (0.5$10 \mathrm{keV}$ ) led to the development of the ABRIXAS mission which unfortunately failed. The optical system is a bundle of seven Wolter I telescopes (each consisting of 27 nested gold-coated mirror shells) titled with respect to each other, focussing images on a single pn CCD (Trümper et al. 1998; Predehl 1999). The on-axis HEW is about 20" (Predehl 1999 ) degrading to $\sim 80^{\prime \prime}$ at $20^{\prime}$. The resulting mean resolution is about $40^{\prime \prime}$. A rough positional accuracy estimate can be derived by the ratio $\mathrm{HEW} /(2 \sqrt{N-1})$ (e.g. Lazzati et al. 1998, since for a 2-dimensional Gaussian the HEW coincides with the $2 \sigma$ ) where $N$ the number of photons. So for a 10 photons detection limit a positional accuracy of $\sim 12^{\prime \prime}$ is achieved (90\% confidence level).

The main driver of the ABRIXAS mission and in general of sky surveys at energies higher than $2 \mathrm{keV}$ was the study of the extragalactic background and of absorbed Active Galactic Nuclei (AGN). Moreover, clusters of galaxies are among the best tracers of the large-scale structure and their evolution is a powerful diagnostic for the geometry of the universe (e.g. Borgani \& Guzzo 2001). Clusters at any redshift and cosmology can be detected as extended sources if the HEW is better than $\sim 15^{\prime \prime}$. In a flux-limited survey down to $\sim 10^{-14} \mathrm{erg} \mathrm{s}^{-1} \mathrm{~cm}^{-2}$, clusters of galaxies will be detected in large numbers up to $z \sim 1$ and AGN up to $z \sim 4$. With that good angular resolution, clusters of galaxies can be detected and picked up directly in the $\mathrm{X}$-ray images as extended sources.

A more efficient way to carry out the mapping of the $\mathrm{X}$-ray sky is to use an optimized mirror system with a large corrected field of view. We consider here a Surveying $\mathrm{X}$-ray Telescope (SXT) made by optimized mirrors with an effective area comparable to that of the Chandra instrument, i.e. $A_{\text {eff }} \sim 600 \mathrm{~cm}^{2}$ at $1.5 \mathrm{keV}$. This number includes the mirror effective area, the CCD quantum efficiency and filter transmission. We consider here CCDs like the EPIC/MOS on board XMM-Newton or the ACISI on board Chandra. These are characterized by a small pixel size but by a reduced response at high $(>4 \mathrm{keV})$ energies, at variance with the EPIC-pn CCD adopted by ABRIXAS. Iridium coating of the mirrors will improve the response of the SXT at high energies providing a total effective area at $6 \mathrm{keV}$ of $\sim 130 \mathrm{~cm}^{2}$. For an outermost shell diameter of $70 \mathrm{~cm}$ diameter, we would need 50 mirror 
Table 5. All-sky surveys with existing and planned X-ray telescopes.

\begin{tabular}{ccc|c}
\hline & ROSAT & ABRIXAS & SXT \\
\hline Energy range $(\mathrm{keV})$ & $0.1-2.4$ & $0.3-10$ & $0.3-10$ \\
Area @ 1.5 keV $\left(\mathrm{cm}^{2}\right)$ & 200 & 80 & 600 \\
HEW on-axis $(\operatorname{arcsec})$ & 20 & 20 & 10 \\
Average HEW over field of view & 60 & 40 & 10 \\
Solid angle $\left(\mathrm{deg}^{2}\right)$ & 3 & 2.4 & 1.4 \\
Average exposure time $(\mathrm{s})$ & 500 & 4,000 & 2,000 \\
Time for all-sky (yr) & 0.5 & 3 & 3.0 \\
Indicative positional accuracy $(1 \sigma)^{\dagger}$ & $25^{\prime \prime}$ & $12^{\prime \prime}$ & $3^{\prime \prime}$ \\
\hline Limiting flux (cgs) $(0.5-2 \mathrm{keV})$ & $5 \times 10^{-13}$ & $1 \times 10^{-13}$ & $1 \times 10^{-14}$ \\
Limiting flux (cgs) $(2-10 \mathrm{keV})$ & - & $4 \times 10^{-13}$ & $1 \times 10^{-13}$ \\
\hline
\end{tabular}

For SXT we consider 50 mirror shells reaching an effective area of $600 \mathrm{~cm}^{2}$ at $1.5 \mathrm{keV}$ (after convolution with the CCD and filter responses). The sensitivity has been computed requiring that at least 5 photons are collected within the detection cell and adopting the Chandra background of $0.3 \mathrm{ct} \mathrm{s}^{-1} \mathrm{chip}^{-1}=3 \times 10^{-7} \mathrm{ct} \mathrm{s}^{-1}$ pixel $^{-1}$.

$\dagger$ Accuracy of the Point Speread Function centroid position at the limiting flux level.

shells to reach the selected area. The corrected field with an $\mathrm{HEW}<20^{\prime \prime}$ extends up to $\sim 40^{\prime}$. In order to cover the focal plane, 9 CCDs are needed. These should be displaced in a way similar to the EPIC-MOS CCDs (see above) in order to follow the curved focal plane.

As a second parameter we set the time to carry out an all-sky survey as 3 years. Assuming a field of view of $40^{\prime}$ radius and allowing for a $20 \%$ superposition of different scans we end up with a $2000 \mathrm{~s}$ mean observing time per field. For the computation of the survey sensitivity we assume the background of the Chandra ACIS-I $\left(3 \times 10^{-7}\right.$ ct s$^{-1}$ pixel $\left.^{-1}\right)$, an absorbed power law (photon index 2 and column density of $3 \times 10^{20} \mathrm{~cm}^{-2}$ ) for the source spectrum and a source detection with 5 photons in the detection cell. This last condition is motivated by the very low background in the small detection cell $(\lesssim 0.09$ counts in $2000 \mathrm{~s}$ per detection cell), resulting in a $\sim 5.3 \sigma$ detection. With these constraints we obtain a limiting $0.3-10 \mathrm{keV}$ flux of $2 \times 10^{-14} \mathrm{erg} \mathrm{s}^{-1} \mathrm{~cm}^{-2}$. The flux limit reduces to $1 \times 10^{-14}$ and $1 \times 10^{-13} \mathrm{erg} \mathrm{s}^{-1} \mathrm{~cm}^{-2}$ in the $0.5-2$ and $2-10 \mathrm{keV}$ energy bands, respectively (see Table 5). An indicative positional accuracy at the detection limit of $\lesssim 5^{\prime \prime}$ (90\% c.l.) can be reached.

Such a survey would be the analogue of the Palomar survey in the X-ray and would be useful for the next generation of X-ray satellites, XEUS and Constellation- $X$, as well as to pinpoint places where NGST could image the earliest phases of the forming structure in the Universe.

\section{Conclusions}

$\mathrm{X}$-ray surveys can be more efficiently carried out with mirrors optimized over a large field of view. The polynomial design is superior with respect to the Wolter I design. We show here that our design provides an improvement in the mean HEW over the entire field of view of $\gtrsim 150 \%$ over the Wolter I design. This makes it possible to carry out sky surveys in a shorter time and/or at deeper levels.

In this paper we describe a refinement of the optimization technique put forward by BBG and Werner (1977).
We are able to improve the design of single mirror shells for wide-field imaging by about $40 \%$ in terms of mean angular resolution (HEW) with respect to the original BBG's design, i.e. source detections at the same significance level can be achieved in about a factor of 2 shorter exposure time.

We compare the performances of a wide-field optimized telescope (SXT) sized to an effective collecting area similar to Chandra, with existing and planned mission surveys. The SXT gains a factor of $\sim 10$ in sensitivity mainly due to the improved angular resolution. Furthermore, current and planned $\mathrm{X}$-ray telescopes fail to provide a deep mapping of the $\mathrm{X}$-ray sky during slews whereas serendipituous surveys have access to only a few percent of the sky. In particular, either Chandra and XMM-Newton fail to provide an all-sky survey at the selected limiting flux in a reasonable time $(\gtrsim 20 \mathrm{yr}$, if pointings of $2 \mathrm{ks}$ are carried out).

Technology requirements are severe for the manufacturing of these optimized shells, even if the experience of Chandra has indicated that mirrors with tiny mechanical tolerances can be made (paying however for a large mirror weight). Despite these caveats, a mirror shell following an earlier optimized design has already been built (Citterio et al. 1998, 1999). These authors have demonstrated that, using an upgraded replication technique, it is possible to produce light-weight carriers in ceramic material (Silicon Carbide, $\mathrm{SiC}$, or Allumina, $\mathrm{Al}_{2} \mathrm{O}_{3}$; instead of nickel carriers used for BeppoSAX, JET-X, Newton-XMM and Swift missions), over which the reflecting material (gold in this case) can be deposited. As a result of this process, a single mirror shell with a HEW $<13^{\prime \prime}$ over a $35^{\prime}$ (radius) field of view has been built and tested (see Fig. 4; Citterio et al. 1999; Ghigo et al. 1999). This demonstrates that current technologies meet the fabrication requirements (especially stiffness, e.g. Citterio et al. 1998) of these optimized mirror designs.

Comparison with current and future $\mathrm{X}$-ray missions shows that only a dedicated mission with an optimized mirror is able to produce in a reasonable time an all-sky 
survey down to a limiting flux of a few $10^{-14} \mathrm{erg} \mathrm{s}^{-1} \mathrm{~cm}^{-2}$ in the $0.5-10 \mathrm{keV}$ energy band.

Acknowledgements. We acknowledge useful discussions with G. Chincarini, G. Ghisellini, G. Pareschi \& G. Tagliaferri. This work was partially supported through ASI grants. We thank the referee (B. Aschenbach) for his comments which improved and sharpened the paper.

\section{References}

Aschenbach, B. 1988, Appl. Opt., 27, 1404

Bavdaz, M., et al. 1999, SPIE, 3766, 82

Borgani, S., \& Guzzo, L. 2001, Nature, 409, 39

Burrows, C. J., Burg, R., \& Giacconi, R. 1992, ApJ, 392, 760 (BBG)

Campana, S., et al. 1998, ApJ, 524, 423

Cash, W., Shealy, D. L., \& Underwood, J. H. 1979, SPIE, 184, 228

Cash, W., Shipley, A., Osterman, S., \& Joy, M. 2000, Nature, 407,160

Chincarini, G., et al. 1999, proceedings of "Observational cosmology", ed. G. Giuricin, M. Mezzetti, \& P. Salucci, ASP Ser., 176, 223

Chincarini, G., et al. 2000, PANORAM-X proposal for ESA Flexi-mission F2/F3 program

Citterio, O., et al. 1991, SPIE, 1343, 145

Citterio, O., et al. 1998, SPIE, 3444, 393

Citterio, O., et al. 1999, SPIE, 3766, 198

Gotthelf, E. V., \& White, N. E. 1997, in proc. X-Ray Imaging and Spectroscopy of Cosmic Hot Plasmas, ed. F. Makino, $\&$ K. Mitsuda, 31

Giacconi, R., et al. 1979, ApJ, 230, 540

Gioia, I., et al. 1990, ApJS, 72, 567
Giommi, P., et al. 1991, ApJ, 378, 77

Ghigo, M., et al. 1999, SPIE, 3766, 207

Harvey, J. E., \& Thompson, P. L. 1999, SPIE, 3766, 173

Jansen, F. A., \& XMM Science Operations Team 2000, AAS, 196,3401

Lazzati, D., et al. 1998, ApJ, 524, 414

Nariai, K. 1987, Appl. Opt., 26, 4428

Nariai, K. 1988, Appl. Opt., 27, 345

Predehl, P. 1999, SPIE, 3765, 172

Serlemitsos, P. J., et al. 1992, in Frontiers of X-ray Astronomy, ed. Y. Tanaka, \& K. Koyama (Tokio: Universal Academy Press), 221

Serlemitsos, P. J., et al. 1995, PASJ, 47, 105

Tananbaum, H. D., White, N. E., Bookbinder, J. A., Marshall, F. E., \& Cordova, F. A. 1999, SPIE, 3765, 62

Trümper, J., Hasinger, G., \& Staubert, R. 1998, AN, 319, 113

Turner, M. J. L., et al. 2001, A\&A, 365, L27

VanSpeybroeck, L. P. 1979, SPIE, 184, 2

VanSpeybroeck, L. P., \& Chase, R. C. 1972, Appl. Opt., 11, 440

Voges, W., \& the MPE-ROSAT Team 1999a, Diffuse thermal and relativistic plasma in galaxy clusters, ed. H. Böhringer, L. Feretti, \& P. Schuecker, Garching, Germany MPE, 1999 Voges, W., et al. 1999b, A\&A, 349, 389

Voges, W., et al. 2000, IAUC N.7432

Weisskopf, M. C., Tananbaum, H. D., VanSpeybroeck, L. P., \& O'Dell, S. L. 2000, SPIE, 4138, in press

Werner, W. 1977, Appl. Opt, 16, 764

White, N. E., Giommi, P., \& Angelini, L. 1994, AAS Meeting 18541.11

Wolter, H. 1952a, Ann. Phys., 10, 94

Wolter, H. 1952b, Ann. Phys., 10, 286

Zimmermann, H.-U. 1994, IAUC N.6102 